\title{
Ballistic magnetoresistance in transition-metal nanocontacts: The case of iron
}

\author{
N. García, $\left.{ }^{a}\right)$ M. Muñoz, and Y.-W. Zhao \\ Laboratorio de Física de Sistemas Pequeños y Nanotecnología, Consejo Superior de Investigaciones \\ Cientificas (CSIC), Serrano 144, E-28006 Madrid, Spain
}

(Received 17 December 1999; accepted for publication 9 March 2000)

\begin{abstract}
This letter shows that the ballistic magnetoresistance of Fe at room temperature and low magnetic fields is ten times smaller than for $\mathrm{Ni}$ and $\mathrm{Co}$. The results are well explained by theory that provides a global understanding for $3 d$ transition metals because, for $\mathrm{Fe}$, the ratio of majority to minority spins at Fermi level is much smaller than for $\mathrm{Ni}$ and $\mathrm{Co}$. The data indicate that conduction is carried out by majority $d$ electrons in the case of Fe, in contrast to what happens for Ni and Co. (C) 2000 American Institute of Physics. [S0003-6951(00)03718-9]
\end{abstract}

Recently, ballistic magnetoresistance (BMR) has been measured at room temperature and small applied magnetic fields in nanometer magnetic contacts. ${ }^{1,2}$ Data show that the BMR values for the smaller contacts, approximately $1 \mathrm{~nm}$ size, can reach $200 \%-300 \%$ and then decrease very quickly as the contact size increases. These large values can be explained ${ }^{2-4}$ by Zeeman splitting theory as well as by tightbinding calculations. ${ }^{5}$ The basic physics underlying the experiments is that BMR at the nanocontacts is a nonadiabatic process in the sense that the spin does not have time to flip in the case of an antiferromagnetic configuration on both sides of the nanocontact (see Ref. 1 for details) — the domain-wall boundary/width (DWW) of nanometer size is very sharp. ${ }^{2,6}$ Then, if the ratio $r=D_{\uparrow} / D_{\downarrow}$ (where $D_{\uparrow}$ and $D_{\downarrow}$ are the density of states of majority and minority spins at Fermi level $\left.E_{f}\right)$ is large, the electrons $\uparrow$ on the left side of the nanocontact cannot be accommodated as electrons $\downarrow$ on the right side, and thus suffer strong scattering. However, for the ferromagnetic configuration, the electrons can be accommodated easily on both sides of the contact. As a result, the difference of resistance in both configurations causes very large magnetoresistance (MR) values for $\mathrm{Ni}$ and Co where $r=12$ and 10, as reported by band-structure calculations. ${ }^{7}$ We note that in bulk materials the DWW is much larger than the mean-free path for spin reversal and, hence, the normal MR is very small, of the order of $1 \%$.

To test the theory further, we have chosen to study $\mathrm{Fe}$, since here $r=3,{ }^{7}$ giving a much smaller BMR. In fact, the large values of $r$ for $\mathrm{Ni}$ and Co caused us to perform our first BMR experiments on them.

We proceed now by describing the BMR results for Fe. The samples are high-purity $(99.99+)$ polycrystalline iron rods of $2 \mathrm{~mm}$ diam and $25 \mathrm{~mm}$ length, with tips on opposing ends. In order to provide rigidity and stability to the contact, the iron wires are firmly held by resin in a Teflon tube. The sample electrodes are ultrasonically cleaned in acetone and then in methanol.

Experiments have been performed using the same technique as described in Ref. 1, at room temperature and at maximum applied fields of 120 Oe. Figure 1 shows the data

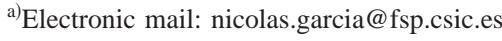

for the magnetoconductance as a function of the number of quantum channels $\left(2 e^{2} / h\right)$. The data show values of $25 \%$ for $\sim 1$ channel, decreasing exponentially and then varying very slowly as $\alpha / N$. This is well described by the Zeeman splitting theory ${ }^{2-4}$ and by tight-binding calculations. ${ }^{5}$ We believe that for observing the BMR effect, there should not be much difference considering what material is used as the nanocontact, as far as it being ballistic: metallic or insulating. ${ }^{8}$ What matters is the magnetic configuration on both sides of the
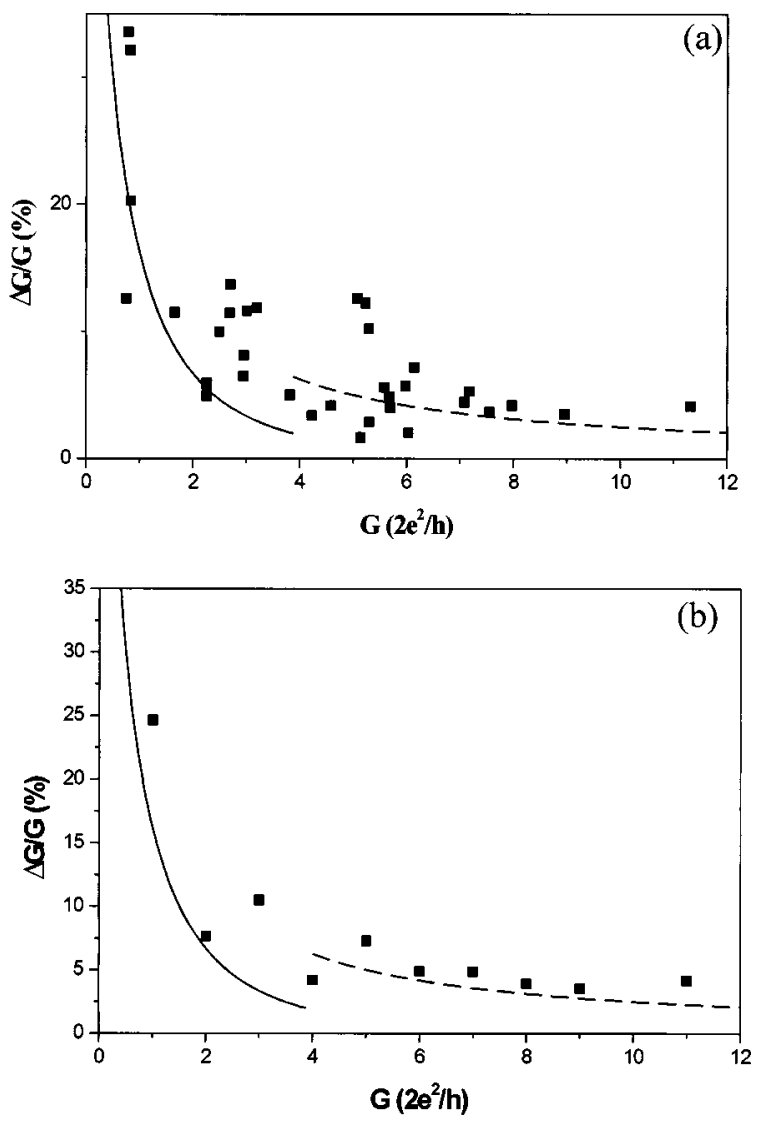

FIG. 1. (a) Experimental values of BMR for iron. The solid line is the calculation for theory developed in Ref. 2 for $\zeta=0.5$ and $r=3$ (from Ref. 7). The dashed line shows the $\alpha / N$ behavior $(\alpha=25)$ for $N \gg 1$. (b) The same as (a) but for the average experimental data. Notice that the BMR values are approximately ten times smaller for Fe than for Ni and Co (in Fig. 2). 


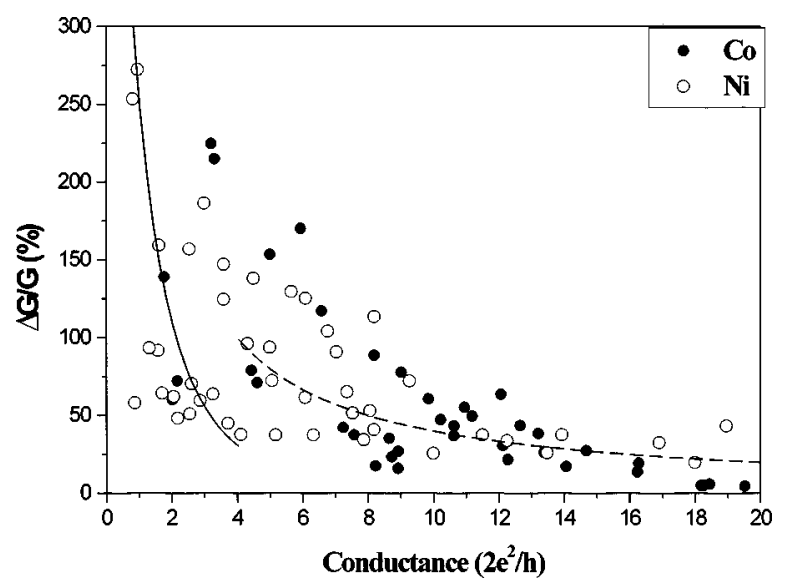

FIG. 2. Ballistic magnetoresistance for $\mathrm{Ni}$ and $\mathrm{Co}$ : the solid line is the calculation for $\zeta=0.87$ and $r=12$; the dashed line shows $\alpha / N$ behavior $(\alpha=400)$. Data for comparison are from Refs. 1 and 2.

nanocontact and the $D_{\uparrow} / D_{\downarrow}$ ratio. For comparison, we present in Fig. 2 the results for Ni and Co from Ref. 2, which show much larger values.

From Ref. 2, the theoretical value of BMR for small contacts $N \sim 1$ is given by

$$
\begin{aligned}
& \operatorname{BMR}(N \sim 1) \propto \frac{\zeta^{2}}{1-\zeta^{2}}, \\
& \zeta=\frac{D_{\uparrow} / D_{\downarrow}-1}{D_{\uparrow} / D_{\downarrow}+1},
\end{aligned}
$$

with $\zeta_{\mathrm{Ni}}=0.87, \zeta_{\mathrm{Co}}=0.83$, and $\zeta_{\mathrm{Fe}}=0.50$, as obtained from band-structure calculations. $^{7}$

The ratio $\operatorname{BMR}(N \sim 1)_{\mathrm{Ni}, \mathrm{Co}} / \mathrm{BMR}(N \sim 1)_{\mathrm{Fe}} \sim 7.2$ agrees well with the experimental value $\sim 8$. The values of $\alpha=25$ for $\mathrm{Fe}$ and 400 for $\mathrm{Ni}$ and $\mathrm{Co}$ also reflect the factor $r$ of the density of states.

It should be stressed that we have used the integrated density of states at $E_{f}$ (Ref. 7) and it could be argued that the values of $r$ may depend on the orientation of the crystallite faces connected by the nanocontact. However, our data represent many contacts, and thus several oriented crystallites may play a role. Therefore, the average values, Fig. 1(b), may be a more suitable set to compare with theory.

Recently, in another experiment ${ }^{9}$ Oberli et al. have injected polarized $s$ electrons $4-7 \mathrm{eV}$ above $E_{f}$ into magnetic layers. The experiments showed that the transmitivity, when the polarization of the ferromagnetic layer is the same with that one of the incident $s$ electrons, is ten times larger than the transmitivity when the polarization of the layer is antiparallel (see Fig. 1 in Ref. 9). These are similar experiments to the one we performed and can be explained by taking $\zeta$ $=0.99$, implying that at the energies of the incident electrons the density of minority electrons is practically zero. This may be shown by inverse polarized photoemission.

In conclusion, we have shown that the BMR in Fe nanocontacts shows percentage values ten times smaller than for $\mathrm{Ni}$ and $\mathrm{Co}$, and theory ${ }^{2,5}$ describes well this behavior due to the much smaller value of the density of states ratio $r$ for $\mathrm{Fe}$ with respect to $\mathrm{Ni}$ and $\mathrm{Co}$. Our data seem to confirm that conduction is carried out by majority $d$ electrons in the case of $\mathrm{Fe}$, in contrast to what happens for $\mathrm{Ni}$ and $\mathrm{Co}$. In closing, we mentioned that in the case of one-band magnets ( $r$ $\rightarrow \infty$ ) the MR should tend to infinity even at room temperature if the transport is ballistic and nonadiabatic for very sharp domain walls. Such a material would also require a mean-free path larger than the nanocontact size for the transport to be ballistic. Also, there could be ferromagnets with a larger magnetic moment but with a very small BMR because $r \sim 1$. Efforts are oriented in this direction.

This work has been supported by the Spanish DGICyT.

${ }^{1}$ N. García, M. Muñoz, and Y.-W. Zhao, Phys. Rev. Lett. 82, 2923 (1999).

${ }^{2}$ G. Tatara, Y.-W. Zhao, M. Muñoz, and N. Garcia, Phys. Rev. Lett. 83, 2030 (1999); also, Alpine Meeting on Giant Magnetoresistance, Obeersdorf, Austria, 1999.

${ }^{3}$ G. G. Cabrera and L. M. Falicov, Phys. Status Solidi B 61, 539 (1974).

${ }^{4}$ G. Tatara and H. Fukuyama, Phys. Rev. Lett. 78, 3773 (1997).

${ }^{5}$ J. Mathon, Phys. Rev. B 56, 11810 (1997).

${ }^{6}$ P. Bruno, Phys. Rev. Lett. 83, 2425 (1999).

${ }^{7}$ J. L. Moruzzi, J. F. Janak, and A. R. Williams, Calculated Band Structure Properties (Pergamon, New York, 1978).

${ }^{8}$ N. Garcia, M. Muñoz, H. Rohrer, and Y.-W. Zhao, Spanish Patent No. P9802091, and international PCT application, 1998.

${ }^{9}$ D. Oberli, R. Burgermeister, S. Riesen, W. Weber, and H. C. Siegmann, Phys. Rev. Lett. 81, 4228 (1998). 